\title{
Simulations of gas puff effects on edge density and ICRF coupling in ASDEX upgrade
}

using EMC3-Eirene

W. Zhang', T. Lunt, V. Bobkov, J.-M. Noterdaeme, D. Coster, P. Jacquet, D. Brida, Y. Feng, and the ASDEX Upgrade Team

Citation: AIP Conference Proceedings 1689, 050006 (2015); doi: 10.1063/1.4936494

View online: http://dx.doi.org/10.1063/1.4936494

View Table of Contents: http://aip.scitation.org/toc/apc/1689/1

Published by the American Institute of Physics 


\title{
Simulations of gas puff effects on edge density and ICRF coupling in ASDEX Upgrade using EMC3-Eirene
}

\author{
W. Zhang ${ }^{1,2,3, a)}$, T. Lunt ${ }^{2}$, V. Bobkov ${ }^{2}$, J-M. Noterdaeme ${ }^{1,2}$, D. Coster ${ }^{2}$, P. Jacquet ${ }^{4}$, \\ D. Brida ${ }^{2}$, Y. Feng ${ }^{5}$, and the ASDEX Upgrade Team ${ }^{2}$ \\ ${ }^{1}$ Applied Physics Department, University of Ghent, Ghent, Belgium \\ ${ }^{2}$ Max-Planck-Institut für Plasmaphysik, Garching, Germany \\ ${ }^{3}$ Institute of Plasma Physics, Chinese Academy of Sciences, Hefei, P. R. China \\ ${ }^{4}$ CCFE, Culham Science Centre, Abingdon, UK \\ ${ }^{5}$ Max-Planck-Institut für Plasmaphysik, Greifswald, Germany \\ ${ }^{a)}$ Corresponding author: wei.zhang@ipp.mpg.de
}

\begin{abstract}
Simulations were carried out with the 3D plasma transport code EMC3-EIRENE, to study the deuterium gas $\left(\mathrm{D}_{2}\right)$ puff effects on edge density and the coupling of Ion Cyclotron Range of Frequency (ICRF) power in ASDEX Upgrade. Firstly we simulated an inter-ELM phase of an H-mode discharge with a moderate $\left(1.2 \times 10^{22}\right.$ electrons/s $)$ lower divertor gas puff. Then we changed the gas source positions to the mid-plane or top of machine while keeping other conditions the same. Cases with different mid-plane or top gas valves are investigated. Our simulations indicate that compared to lower divertor gas puffing, the mid-plane gas puff can enhance the local density in front of the antennas most effectively, while a rather global (toroidally uniform) but significantly smaller enhancement is found for top gas puffing. Our results show quantitative agreement with the experiments.
\end{abstract}

\section{INTRODUCTION}

Ion Cyclotron Range of Frequency (ICRF) heating is one of the main auxiliary plasma heating methods in tokamaks. It relies on the Fast Wave (FW) to transport the power from the edge where the antenna is located to the plasma center. Since the FW is evanescent below the cut-off density (typically in the $10^{18} \mathrm{~m}^{-3}$ range), the wave decays rapidly in the region where the density is below this value in the Scrape-Off Layer (SOL). The coupling depends strongly on the width of this evanescence region. The distance between the ICRF antenna and the FW cutoff layer can be made smaller by increasing the edge density in front of the ICRF antenna.

Previous experiments on ASDEX Upgrade (AUG) [1, 2], JET [3, 4] and DIII-D [5, 6] show that the edge density could indeed be increased with top or mid-plane deuterium gas puffing. A factor 2 increase of antenna loading in these tokamaks was reported for the antennas close to the mid-plane valves in [7].

In order to understand and quantitatively predict the required amounts of gas and to optimize the spatial location of the valves, numerical simulations are required. Due to the assumptions of toroidal axisymmetry, 2D codes such as EDGE2D-EIRENE cannot quantitatively reproduce the experimental results [7]. EMC3-EIRENE is a coupled Edge Monte Carlo 3D plasma fluid (EMC3) and kinetic neutral particle (EIRENE) code [8]. The 3D nature of the code makes it particularly suitable for studying the 3D physics such as gas puffing effects on ICRF power coulping. By including the toroidal nonaxisymmetrical Plasma Facing Components (PFCs) and the gas valves at the correct 3D positions, the simulations can be made more realistic. We report the first simulation results on an inter-ELM phase of an H-mode discharge with different gas puff locations in AUG. Once the model is validated against experiments, it can be applied to ITER and other future fusion devices in order to predict the optimum gas valve positions for maximized ICRF power coupling. 


\section{VALIDATION WITH EXPERIMENTS}

A full $360^{\circ}$ computation grid (Fig. 1) was implemented in our simulations, with the SOL region extended to the wall. We made use of the EMC3-Eirene setup including both the divertor and the Main Chamber (MC) 3D wall PFCs from previous EMC3-Eirene simulations by Lunt et al. [9], and extended the computation model to full $360^{\circ}$. The PFCs including the inner heat shield, 4 auxiliary limiters and 8 ICRF antenna limiters. For the convenience of calculations the toroidal angle is counted from $-180^{\circ}$ to $180^{\circ}$. The $0^{\circ}$ position is chosen at the boundaries of sector 5 and sector 6 .

The AUG discharge used for our simulations is \#31269 (at 2.1s), an ELMy H-mode plasmas with lower divertor gas puff rate $\sim 1.2 \times 10^{22}$ electrons/s. The total heating power is $7.76 \mathrm{MW}$ (NBI $4.89 \mathrm{MW}$, OH $0.21 \mathrm{MW}$, ICRF 1.56MW and ECRH 1.1MW) with 3.17MW radiated power. In our simulations no impurities are considered, hence the radiated power is subtracted from the total power to approximate the total power deposited on the PFCs by the plasma. The separatrix density is about $2.5 \times 10^{19} \mathrm{~m}^{-3}$.

The mid-plane profiles $n_{e}, T_{e}$ and divertor profile $j_{\text {sat }}$ in the code are fitted to the experiment by modifying the tranport parameters (i. e. particle and heat diffusion coefficient $\mathrm{D}_{\perp}$ and $\mathrm{x}_{\perp}$ respectively) in the code. The ELMs were filtered out from the experimental data, because the inter-ELM phases are those of interest corresponding to the lowest coupling resistance and maximum antenna voltages [7]. The diagnostics used for comparison include the Lithum beam for electron density, edge and core Thomson scattering for electron density and temperature, and Langmuir probes for the particle flux to the targets. With the input transport parameters as shown in Fig. 2, the final mid-plane profiles (density and temperature) and divertor profiles (particle fluxes to the targets) in our simulations agree well with the experiments, except for the $j_{\text {sat }}$ values at the inner strike point.

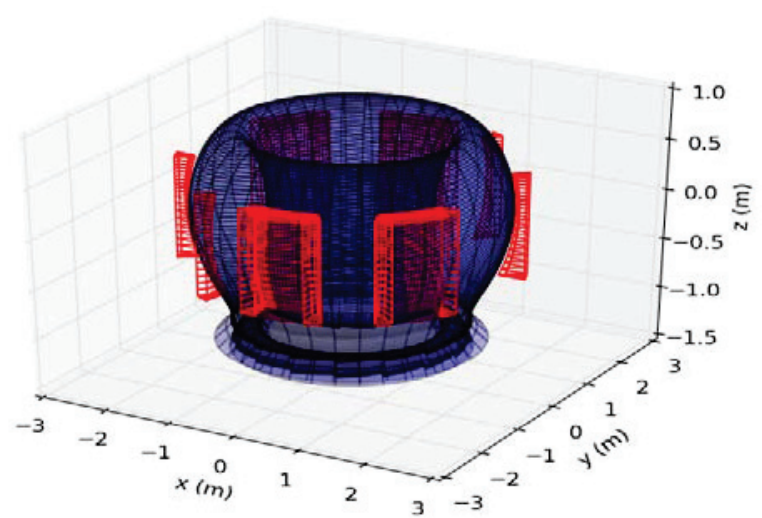

FIGURE 1. The full grid with 8 ICRF limiters (two on both sides of each ICRF antenna) and 4 auxiliary limiters.

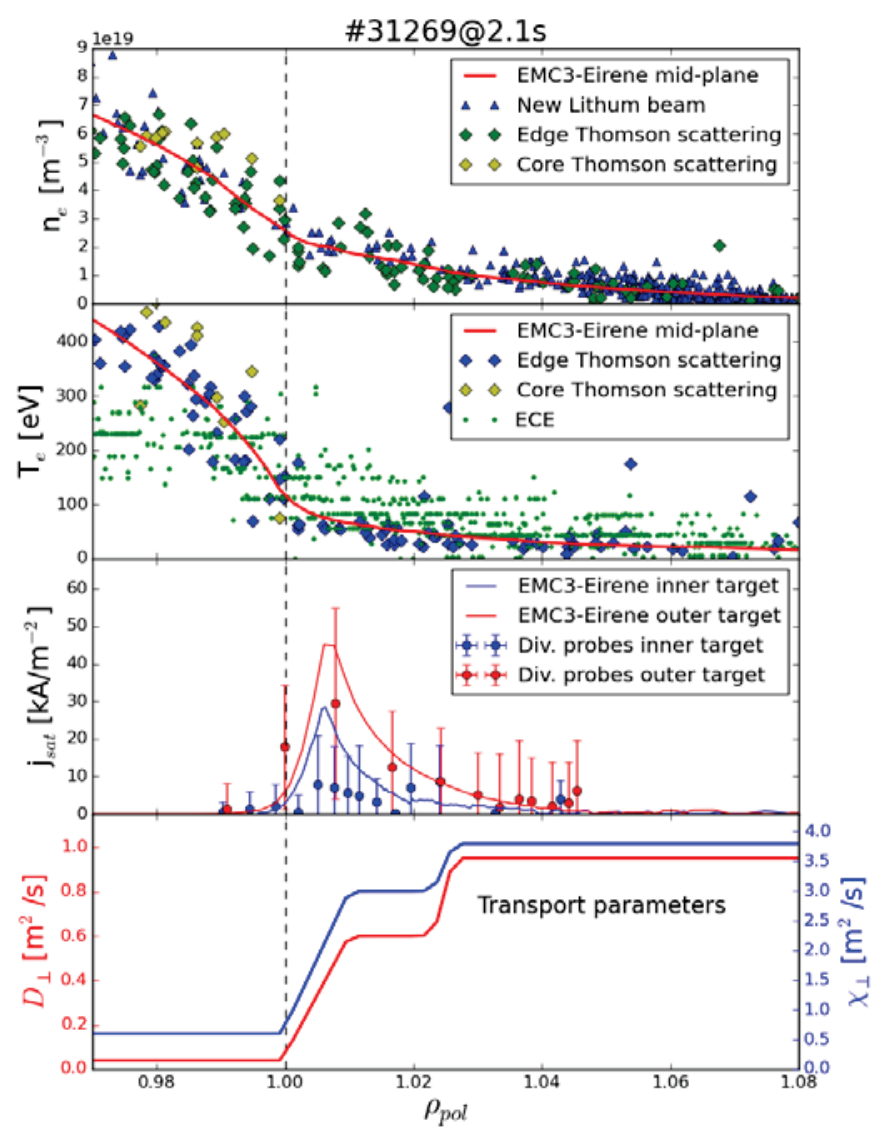

FIGURE 2. EMC3-Eirene simulations compared to experimental diagnostics.

\section{GAS PUFFING EFFECTS ON EDGE DENSITY}

For experiments in AUG, usually 4 gas valves are used for lower divertor gas puffing and one for top or midplane gas puffing. The following cases were simulated: I. four lower divertor gas valves in ports 1, 5, 9, 13 (toroidal angle $\left.\varphi_{\mathrm{v}}=-101.25^{0},-11.25^{\circ}, 78.75^{\circ}, 168.75^{\circ}\right)$; II. one top gas valve in port $2\left(\varphi_{\mathrm{v}}=-78.75^{\circ}\right)$; III. one top gas valve in port $7\left(\varphi_{\mathrm{v}}=33.75^{0}\right)$; IV. one mid-plane gas valve in port $3\left(\varphi_{\mathrm{v}}=-56.25^{0}\right)$; V. one mid-plane gas valve in port 13 $\left(\varphi_{\mathrm{v}}=168.75^{0}\right)$. The total gas puff rates in all cases are the same $\left(1.2 \times 10^{22}\right.$ electrons/s $)$. 
Note that the mid-plane gas valves are located very deep in the port (a so-called A-port) in AUG. Before the gas reaches the plasma edge, it has already spread widely and becomes an almost homogeneous source in the crosssection of the port. The scattered gas is firstly shaped by the front face of the port and then by the PFCs such as limiters. In the simulations, we have put the mid-plane gas valves as far as possible at the edge of the computational grid. In that way the gas fills-up the A-port. This gives a realistic description of the ionization area where neutrals interact with the plasma.

Poloidal cross-sections of the neutral atom densities during lower divertor, top and mid-plane gas puffing are shown in the upper row of Fig. 3. Toroidal positions are chosen where the gas valves are placed, so the cross-section plots are the same either for the top gas puff case II $\left(\varphi=\varphi_{\mathrm{v}}=-78.75^{\circ}\right)$ and case III $\left(\left(\varphi=\varphi_{\mathrm{v}}=33.75^{\circ}\right)\right.$, or for the mid-plane gas puff case IV $\left(\left(\varphi=\varphi_{\mathrm{v}}=-56.25^{\circ}\right)\right.$ and case $\mathrm{V}\left(\left(\varphi=\varphi_{\mathrm{v}}=168.75^{\circ}\right)\right.$. The arrows in the figure are used to represent the positions and directions of the injected gas. A localized cloud of neutrals near the valves could be seen both in the top and the lower divertor gas puff cases; for mid-plane gas puffing the poloidal and toroidal distribution of the enhanced neutral pressure essentially corresponds to the A-port footprint. The corresponding electron densities are also depicted in the lower row of Fig. 3. The densities in the lower divertor and core are the same for all gas puffing cases. A localized density near the top gas valve could be seen, due to the fact that the particle velocities in the parallel direction of the magnetic field are much larger than in the perpendicular one. For midplane gas puffing, the density is enhanced in a large poloidal range in the outer mid-plane; the striped density structures in the top of the SOL are consequences of magnetic field lines which connect the midplane and top in a helical path.

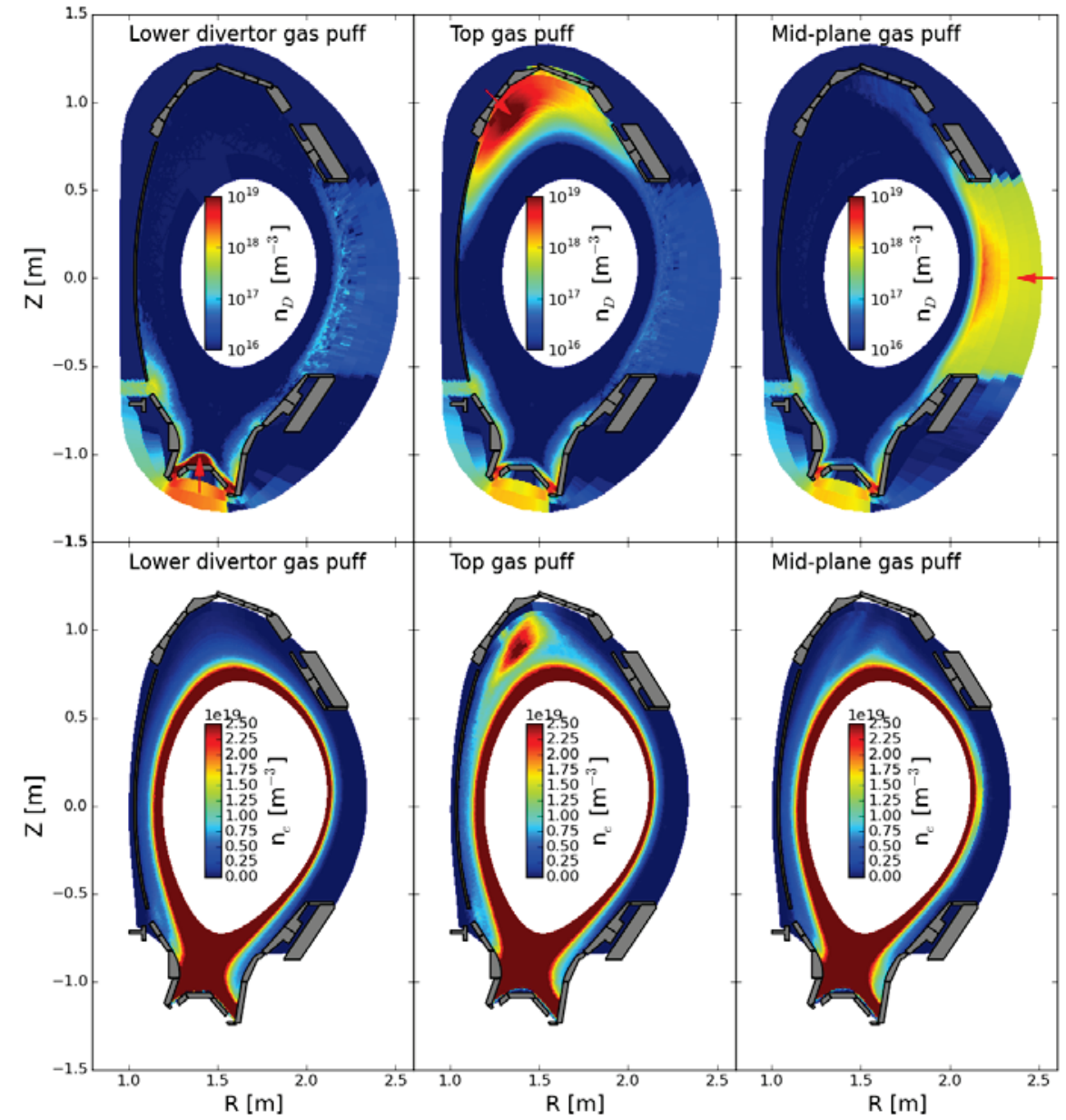

FIGURE 3. Neutral atom and electron densities in the poloidal cross sections for lower divertor, top and mid-plane gas puff cases.

Furthermore, we calculated the averaged electron density both in poloidal and toroidal directions in front of all the ICRF antennas. The comparisons of the averaged density profiles in the cases II, IV, V with case I are given in Fig. 4 (the results of top gas puff cases II and III are the same). In each subplot of Fig. 4, the dashed line represents the density profile which is toroidally uniform during lower divertor gas puffing (case I), and the dot-dashed line is the cut-off density of the ICRF FW in AUG. From the analysis we find that top gas puffing can increase the edge desity to a small extent, but this increase is toroidally uniform and is independent of the toroidal positions of the gas valve (Fig. 4(a)). This can be explained by the field line topology in these discharges [7]. Field lines from the top encounter a large toroidal spread toward the outer-mid-plane which explains the evenly toroidally distributed density at the outer mid-plane when puffing from top. The mid-plane gas puff can significantly increase the edge density in the area near the valve. In the case IV, the gas valve is located between antenna 1 and 2 (closer to antenna 2) and far 
away from antenna 3 and 4, and the density is enhanced largely in front of antennas 1 and 2, while almost no change is observed for antennas 3 and 4 (Fig. 4(b)). In the case V, the toroidal distances between antennas 4, 3, 1, 2 and the valve are increasing, and the density enhancement has the inverse trend, i.e. the largest density increase is seen for antenna 4 and the smallest increase for antenna 2 (Fig. 4(c)). The top gas puff cases II or III can reduce the FW cut-off layer and ICRF antenna distance by $\sim 0.73 \mathrm{~cm}$, while the mid-plane gas puff can have a larger effect, such as case IV can make this distance smaller by $\sim 1.96 \mathrm{~cm}$. Based on our results and further calculations with FELICE, the relative improvement of resistances from simulations are in good agreement with the experimental ones [10].

FIGURE 4. Comparisions of averaged densities in front of the antennas between top and lower divertor gas puffs, mid-plane and lower divertor gas puffs.

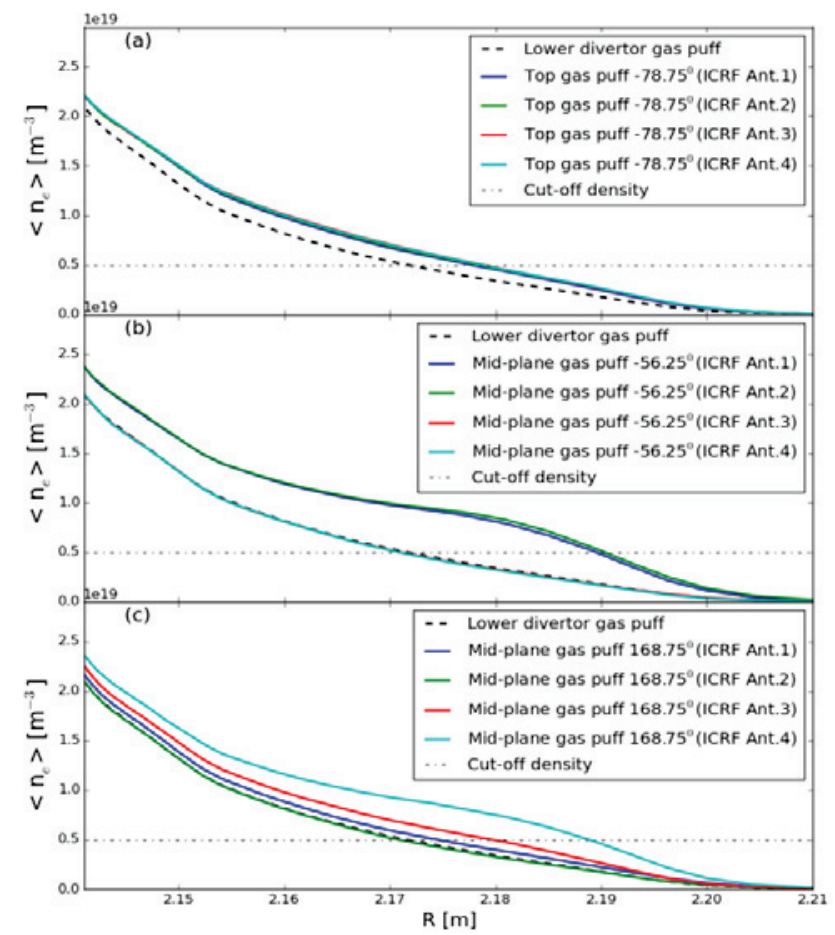

\section{CONCLUSIONS AND OUTLOOK}

For the first time EMC3-Eirene simulations for AUG with a full $360^{\circ}$ grid including MC PFCs, divertor and 3D gas puffs were carried out. Our simulation results are in quantitative agreement with the experiment: Top gas puffing can increase the edge density uniformly in toroidal direction to a small extent, independent of the toroidal position of the top valves. For the mid-plane gas puff the enhancement of the edge density is most significant near the valve and decreases gradually in the toroidal direction; the largest increase is found in front of the antenna nearest to the valve, and almost no change is observed for antennas far away. Thus for ICRF coupling purposes, if poloidal localization of the density increase is not problematic, poloidally distributed gas valves (covering the height of the antennas) which are close to the ICRF antennas in the toroidal direction is the better option.

In the near future we will have better comparison with experiments as more toroidal distributed reflectometers are being mounted in AUG. Further EMC3-Eirene simulations and a comparison with experiments in L and $\mathrm{H}$ mode discharges in AUG, will allow optimization of the gas valve positions in AUG for the purpose of ICRF power coupling and reliable benchmarking of the 3D code in view of its use for ITER. We plan to carry on similar simulations for ITER including the divertor/PFCs/gas valves configurations in full $360^{\circ}$ geometry.

Acknowledgments: This work has been carried out within the framework of the EUROfusion Consortium and has received funding from the Euratom research and training programme 2014-2018 under grant agreement No 633053. The views and opinions expressed herein do not necessarily reflect those of the European Commission.

\section{REFERENCES}

1. V. Bobkov, et al., AIP Conf. Proc. 1580, 271 (2014)

2. P. Jacquet, et al., Nuclear Fusion 52 (2012) 042002

3. E. Lerche, et al, J. Nucl. Mater. (2014), http://dx.doi.org/10.1016/i.jnucmat.2014.10.074 in press.

4. M.-L. Mayoral et al., AIP Conf Proc. 933 (2007) p.55

5. Pinsker R.I., et al., 37th EPS Conf. (2010) http://ocs.ciemat.es/EPS2010PAP/pdf/O4.124.pdf

6. Pinsker R.I., et al., AIP Conf Proc. 1406 (2011) p.313.

7. P. Jacquet et al., Proc. 25th Int. Conf. on Fusion Energy (St. Petersburg, Russia, 2014), EX/P5-39.

8. Y. Feng, et al., Contrib. Plasma Phys. 44 (2004) No. 1-3, 57-69.

9. T. Lunt et al., J. Nucl. Mater. (2014), http://dx.doi.org/10.1016/j.jnucmat.2014.09.020

10. V. Bobkov et al., this conference. 